\title{
ON TWO ABSOLUTE RIESZ SUMMABILITY FACTORS OF INFINITE SERIES
}

\author{
MEHMET ALI SARIGÖL
}

(Communicated by Andrew M. Bruckner)

\begin{abstract}
This paper gives a necessary and sufficient condition in order that a series $\sum a_{n} \varepsilon_{n}$ should be summable $\left|R, q_{n}\right|$ whenever $\sum a_{n}$ is summable $\left|R, p_{n}\right|_{k}, k \geq 1$, and so extends the known result of Bosanquet to the case $k>1$.
\end{abstract}

\section{INTRODUCTION}

Let $\sum a_{n}$ be a given infinite series with $s_{n}$ its $n$th partial sum, and let $\left(p_{n}\right)$ be a sequence of positive real numbers such that $P_{n}=p_{0}+p_{1}+\cdots+p_{n} \rightarrow \infty$ as $n \rightarrow \infty$. If

$$
\sum_{n=1}^{\infty} n^{k-1}\left|t_{n}-t_{n-1}\right|^{k}<\infty
$$

where $t_{n}$ denotes Riesz mean of $\sum a_{n}$, i.e.,

$$
t_{n}=P_{n}^{-1} \sum_{v=0}^{n} p_{v} s_{v}
$$

then the series $\sum a_{n}$ is said to be summable $\left|R, p_{n}\right|_{k}, k \geq 1$. Also $\left|R, p_{n}\right| \equiv$ $\left|R, p_{n}\right|_{1} \equiv\left|\bar{N}, p_{n}\right|$.

\section{MAIN RESUlt}

It is known that the summability $\left|R, q_{n}\right|$ and the summability $\left|R, p_{n}\right|_{k}$, $k \geq 1$, are in general independent of each other. It is, therefore, natural to find out suitable summability factors $\left(\varepsilon_{n}\right)$ so that $\sum a_{n} \varepsilon_{n}$ may be summable $\left|R, p_{n}\right|_{k}$ whenever $\sum a_{n}$ is summable $\left|R, q_{n}\right|$, and, conversely, if $\sum a_{n}$ is summable $\left|R, p_{n}\right|_{k}$ then $\sum a_{n} \varepsilon_{n}$ may be summable $\left|R, q_{n}\right|$. In a paper [4] the author has examined the summability factors problem of the first type. We propose to study the converse problem in the present paper. In what follows we shall prove the following

Received by the editors April 1, 1991 and, in revised form, September 27, 1991.

1991 Mathematics Subject Classification. Primary 40F05, 40D25, 40 G99.

This paper was supported by TBAG-C2 (TUBIT AK). 
Theorem. The necessary and sufficient condition for a series $\sum a_{n} \varepsilon_{n}$ to be summable $\left|R, q_{n}\right|$ whenever $\sum a_{n}$ is summable $\left|R, p_{n}\right|_{k}, k \geq 1$, are

$$
\left\{m^{-1 / k^{\prime}}\left(\frac{q_{m} P_{m}\left|\varepsilon_{m}\right|}{Q_{m} p_{m}}+\left|\frac{P_{m} \Delta \varepsilon_{m}}{p_{m}}+\varepsilon_{m+1}\right|\right)\right\} \in l_{k^{\prime}},
$$

where $1 / k+1 / k^{\prime}=1$.

It may be remarked that our theorem includes, as a special case $k=1$ and $\varepsilon_{m}=1$, the following theorem of Sunouchi [5] and Bosanquet [2].

Theorem A. The necessary and sufficient condition for a series $\sum a_{n}$ to be summable $\left|\bar{N}, q_{n}\right|$ whenever it is summable $\left|\bar{N}, p_{n}\right|$ is

$$
q_{n} P_{n} / Q_{n} p_{n}=O(1) \text { as } n \rightarrow \infty .
$$

The sufficiency of this theorem was proved by Sunouchi [5].

\section{REQUIRED LEMMAS}

We require the following lemmas for the proof of the theorem.

Lemma 1. Let $p \geq 1, k \geq 1$ and suppose that $x, y, u$, and $v$ are related as

$$
\begin{array}{ll}
y_{n}=\sum_{m=0}^{\infty} c_{n m} x_{m}, & n \geq 0, \\
v_{m}=\sum_{n=0}^{\infty} c_{n m} u_{n}, & m \geq 0 .
\end{array}
$$

The necessary and sufficient condition for $y \in l_{p}$ whenever $x \in l_{k}$ is $v \in l_{k^{\prime}}$ whenever $u \in l_{p^{\prime}}$, where $k^{\prime}$ and $p^{\prime}$ are the conjugate indices of $k$ and $p$, respectively (see [3, Lemma 14]).

Lemma 2. If $k>1$ and $y_{n}=\sum_{m=0}^{n} c_{n m} x_{m}$ and $\sum_{n=0}^{\infty}\left|y_{n}\right|<\infty$ whenever $\sum_{m=0}^{\infty}\left|x_{m}\right|^{k}<\infty$, then $\sum_{n=0}^{\infty}\left|c_{n n}\right|^{k^{\prime}}<\infty$.

This lemma is contained in the corollary to Theorem 10 of [1].

\section{PROOF OF THE THEOREM}

Since the proof is easy for $k=1$, it is omitted. Suppose that $k>1$, and let $\left(t_{n}\right)$ and $\left(T_{n}\right)$ be the sequences of Riesz means $\left(R, p_{n}\right)$ and $\left(R, q_{n}\right)$ of the series $\sum a_{n}$ and $\sum a_{n} \varepsilon_{n}$, respectively. Then by the definition, we have

$$
\begin{aligned}
& t_{n}=\frac{1}{P_{n}} \sum_{v=0}^{n} p_{v} \sum_{r=0}^{v} a_{r}=\frac{1}{P_{n}} \sum_{v=0}^{n}\left(P_{n}-P_{v-1}\right) a_{v}, \quad P_{-1}=0, \\
& T_{n}=\frac{1}{Q_{n}} \sum_{v=0}^{n} q_{v} \sum_{r=0}^{v} a_{r} \varepsilon_{r}=\frac{1}{Q_{n}} \sum_{v=0}^{n}\left(Q_{n}-Q_{v-1}\right) a_{v} \varepsilon_{v}, \quad Q_{-1}=0 .
\end{aligned}
$$

A few calculations reveal that, for $n \geqslant 1$,

$$
x_{n}=t_{n}-t_{n-1}=\frac{p_{n}}{P_{n} P_{n-1}} \sum_{v=1}^{n} P_{v-1} a_{v}, \quad x_{0}=a_{0},
$$


and

$$
y_{n}=T_{n}-T_{n-1}=\frac{q_{n}}{Q_{n} Q_{n-1}} \sum_{v=1}^{n} Q_{v-1} a_{v} \varepsilon_{v}, \quad y_{0}=a_{0} \varepsilon_{0} .
$$

It follows by making use of (4.1) that

$$
\begin{aligned}
y_{n}= & \frac{q_{n}}{Q_{n} Q_{n-1}} \sum_{v=1}^{n} Q_{v-1} \varepsilon_{v}\left(\frac{P_{v}}{p_{v}} x_{v}-\frac{P_{v-2}}{p_{v-1}} x_{v-1}\right) \\
= & \frac{q_{n}}{Q_{n} Q_{n-1}} \sum_{v=1}^{n-1} Q_{v-1} \varepsilon_{v} \frac{P_{v}}{p_{v}} x_{v}+\frac{q_{n} P_{n} \varepsilon_{n}}{Q_{n} p_{n}} x_{n} \\
& -\frac{q_{n}}{Q_{n} Q_{n-1}} \sum_{v=1}^{n} Q_{v-1} \varepsilon_{v} \frac{P_{v-2}}{p_{v-1}} x_{v-1} \\
= & \frac{q_{n}}{Q_{n} Q_{n-1}} \sum_{v=1}^{n-1}\left(\frac{P_{v}}{p_{v}} \Delta\left(Q_{v-1} \varepsilon_{v}\right)+Q_{v} \varepsilon_{v+1}\right) x_{v}+\frac{q_{n} P_{n} \varepsilon_{n}}{Q_{n} p_{n}} x_{n} .
\end{aligned}
$$

Put $X_{n}=n^{1-1 / k} x_{n}$ for $n \geq 1$. Then

$$
\begin{aligned}
y_{n} & =\frac{q_{n}}{Q_{n} Q_{n-1}} \sum_{v=1}^{n-1}\left(\frac{P_{v}}{p_{v}} \Delta\left(Q_{v-1} \varepsilon_{v}\right)+Q_{v} \varepsilon_{v+1}\right) v^{1 / k-1} X_{v}+\frac{q_{n} P_{n} \varepsilon_{n}}{Q_{n} p_{n}} n^{1 / k-1} X_{n} \\
& =\sum_{v=1}^{\infty} c_{n v} X_{v}
\end{aligned}
$$

where

$$
c_{n v}=\left\{\begin{array}{l}
\frac{q_{n}}{Q_{n} Q_{n-1}}\left(\frac{P_{v}}{p_{v}} \Delta\left(Q_{v-1} \varepsilon_{v}\right)+Q_{v} \varepsilon_{v+1}\right) v^{1 / k-1}, \quad 1 \leq v \leq n-1, \\
\frac{q_{n} P_{n} \varepsilon_{n}}{Q_{n} p_{n}} n^{1 / k-1}, \quad v=n \\
0, \quad v>n .
\end{array}\right.
$$

Now $\sum a_{n} \varepsilon_{n}$ is summable $\left|R, q_{n}\right|$ whenever $\sum a_{n}$ is summable $\left|R, p_{n}\right|_{k}$ if and only if

$$
\sum\left|y_{n}\right|<\infty \text { whenever } \sum\left|X_{n}\right|^{k}<\infty .
$$

Using Lemma 1 , the necessary and sufficient conditions for the same are

$$
\sum_{n=v}^{\infty} c_{n v} u_{n} \text { is convergent for every } u_{n}=O(1), \quad v \geq 1,
$$

and

Now

$$
\sum_{v=1}^{\infty}\left|\sum_{n=v}^{\infty} c_{n v} u_{n}\right|^{k^{\prime}}<\infty \quad \text { whenever } u_{n}=O(1)
$$

$$
\begin{aligned}
\sum_{n=v}^{\infty} c_{n v} u_{n} & =\frac{q_{v} P_{v} \varepsilon_{v}}{Q_{v} p_{v}} v^{1 / k-1} u_{v}+\left(\frac{P_{v}}{p_{v}} \Delta\left(Q_{v-1} \varepsilon_{v}\right)+Q_{v} \varepsilon_{v+1}\right) v^{1 / k-1} \sum_{n=v+1}^{\infty} \frac{q_{n} u_{n}}{Q_{n} Q_{n-1}} \\
& =\frac{q_{v} P_{v} \varepsilon_{v}}{Q_{v} p_{v}} v^{1 / k-1} u_{v}+\left(\frac{P_{v}}{p_{v}} \Delta\left(Q_{v-1} \varepsilon_{v}\right)+Q_{v} \varepsilon_{v+1}\right) v^{1 / k-1} \delta_{v}
\end{aligned}
$$


where $\delta_{v}=\sum_{n=v+1}^{\infty}\left(q_{n} / Q_{n} Q_{n-1}\right) u_{n}$, which is convergent, since $u_{n}=O(1)$. Thus, a necessary and sufficient condition for (4.3) is

$$
\sum_{v=1}^{\infty}\left|\frac{q_{v} P_{v} \varepsilon_{v}}{Q_{v} p_{v}} v^{1 / k-1} u_{v}+\left(\frac{P_{v}}{p_{v}} \Delta\left(Q_{v-1} \varepsilon_{v}\right)+Q_{v} \varepsilon_{v+1}\right) v^{1 / k-1} \delta_{v}\right|^{k^{\prime}}<\infty,
$$

whenever $u_{n}=O(1)$.

Necessity. Let $u_{v}=1$ for all $v \geq 1$. Then $\delta_{v}=1 / Q_{v}$. Hence, it follows from (4.4) that

$$
\sum_{v=1}^{\infty}\left\{v^{-1 / k^{\prime}}\left|\frac{P_{v}}{p_{v}} \Delta \varepsilon_{v}+\varepsilon_{v+1}\right|\right\}^{k^{\prime}}<\infty
$$

In addition, we have, by Lemma 2 ,

$$
\sum_{n=1}^{\infty}\left\{n^{-1 / k^{\prime}}\left|\frac{q_{n} P_{n} \varepsilon_{n}}{Q_{n} p_{n}}\right|\right\}^{k^{\prime}}<\infty
$$

Hence, combining (4.5) and (4.6), we get from the inequality

$$
a^{k^{\prime}}+b^{k^{\prime}} \leq(a+b)^{k^{\prime}} \leq 2^{k^{\prime}}\left(a^{k^{\prime}}+b^{k^{\prime}}\right), \quad a, b \geq 0,
$$

that the hypothesis of the theorem is necessary.

Sufficiency. This part of the proof follows immediately from (4.5), (4.6), and the above inequality, since (4.4) holds whenever $u_{n}=0(1)$. Therefore the proof of the theorem is completed.

\section{ACKNOWLEDGMENT}

I wish to express my sincerest thanks to the referee for his helpful comments and suggestions.

\section{REFERENCES}

1. G. Bennett and N. J. Kalton, Inclusion theorems for K-spaces, Canad. J. Math. 25 (1973), 511-524.

2. L. S. Bosanquet, Math. Rev. 11 (1950), 654.

3. W. L. C. Sargent, Some sequence spaces related to the $l^{p}$ spaces, J. London Math. Soc. (2) 35 (1960), 161-171.

4. M. A. Sarigöl, On the absolute Riesz summability factors of infinite series (to appear).

5. G. Sunouchi, Notes on Fourier Analysis, 18, absolute summability of a series with constant terms, Tohoku Math. J. 1 (1949), 57-65.

Department of Mathematics, University of ErciYes, 38039 Kayseri, TURKey

E-mail address: SARIGÖL AT TRERUN.BITNET 